\title{
INFLUÊNCIA DOS PARÂMETROS ABIÓTICOS NA ABUNDÂNCIA DE Biomphalaria straminea (BASOMMATOPHORA: PLANORBIDAE) EM UMA LAGOA TEMPORÁRIA NO SEMIÁRIDO DE PERNAMBUCO, BRASIL
}

\author{
Cicero Diogo Lins de Oliveira ${ }^{1 *}$ \& Girlene Fábia Segundo Viana ${ }^{2}$
}

\begin{abstract}
1 Universidade Federal Rural de Pernambuco, Unidade Acadêmica de Serra Talhada, Laboratório de Dinâmica de Populações Aquáticas, Av. Gregório Ferraz Nogueira, s/n, CEP 56909-535, Serra Talhada, PE, Brasil. ${ }^{2}$ Universidade Federal Rural de Pernambuco, Unidade Acadêmica de Serra Talhada, Laboratório de Bentos, Av. Gregório
Ferraz Nogueira, s/n, CEP 56909-535, Serra Talhada, PE, Brasil.

E-mails: linsdiogoc@gmail.com (*autor correspondente); fabiaviana@yahoo.com.br
\end{abstract}

\begin{abstract}
Resumo: As lagoas temporárias representam ecossistemas de grande importância para a manutenção da diversidade biológica no semiárido brasileiro. O objetivo desse estudo foi avaliar as variáveis abióticas que afetam a abundância de Biomphalaria straminea em uma lagoa temporária no semiárido de Pernambuco. O estudo foi desenvolvido na Lagoa II do Parque Estadual Mata da Pimenteira, PE, Brasil. Cinco pontos na lagoa foram amostrados com arrastos, cinco vezes no período de outubro/2014 a julho/2015, com puçá com malha de $0,5 \mathrm{~mm}$. As variáveis limnológicas de cada ponto foram mensuradas mensalmente. Foram registradas apenas duas espécies de gastrópodes, sendo elas B. straminea (Dunker, 1848) (Basommatophora: Planorbidae) com 97,99\% e Pomacea lineata (Spix, 1827) (Architaenioglossa: Ampullariidae) com 2,01\% dos indivíduos. O índice de abundância de B. straminea foi negativamente relacionado com a temperatura e positivamente relacionado com o oxigênio dissolvido. Esses resultados mostram que, dentre as variáveis medidas, esses fatores abióticos são os principais determinantes da abundância de B. straminea nessa lagoa.
\end{abstract}

Palavras-chave: fatores abióticos; moluscos límnicos; semiárido brasileiro.

INFLUENCE OF ABIOTIC PARAMETERS IN THE ABUNDANCE OF Biomphalaria straminea (BASOMMATOPHORA: PLANORBIDAE) IN A TEMPORARY LAGOON IN THE PERNAMBUCO SEMI-ARID, BRAZIL. Temporary lagoons represent ecosystems of great importance for the maintenance of biological diversity in the Brazilian semi-arid region. The objective of this study was to evaluate the abiotic variables that affect the abundance of Biomphalaria straminea in a temporary lagoon in the semi-arid region of Pernambuco. The study was developed in Lagoa II of Mata da Pimenteira State Park, Pernambuco, Brazil. Five points in the lagoon were sampled with trawls, five times in the period from October/2014 to July/2015, with a $0.5 \mathrm{~mm}$ of knitted. The limnological variables of each point were measured monthly. Only two species of gastropods were recorded: B. straminea (Dunker, 1848) (Basommatophora: Planorbidae) with $97.99 \%$ and Pomacea lineata (Spix, 1827) (Architaenioglossa: Ampullariidae) with $2.01 \%$ of the individuals. The abundance index of $B$. straminea was negatively related with temperature and positively related with dissolved oxygen. These results show that among the variables measured these abiotic factors are the main determinants of the abundance of B. straminea in this lagoon.

Keywords: abiotic factors; limnic molluscs; Brazilian semi-arid. 


\section{INTRODUÇÃO}

A região semiárida brasileira caracteriza-se pela irregularidade hídrica e formação de lagoas temporárias, ecossistemas de grande importância já que consistem em ambientes naturais com acúmulo de água durante o período de chuvas (Simões et al. 2008). As lagoas podem secar na estação seca ou em períodos prolongados de estiagem, embora muitas vezes, mesmo no período chuvoso, as chuvas não sejam capazes de manter os ecossistemas lênticos inundados durante um longo tempo (Diniz et al. 2013).

Particularmente para a região nordeste do Brasil, estes corpos d'água podem apresentar alta biodiversidade (Cardoso et al. 2012), desta forma as lagoas temporárias possuem alta importância para a manutenção da diversidade da fauna e flora nessa região (Maltchik 2000, Tundisi \& Matsumura-Tundisi 2008). No entanto, os processos ecológicos associados à comunidade biológica e ao ambiente que mantêm a diversidade de espécies em níveis local e regional ainda são mal compreendidos, especialmente nos sistemas aquáticos tropicais (Aranguren-Riaño et al. 2011) e para as comunidades da fauna bentônica (Vidigal et al. 2005).

Os moluscos límnicos exercem importantes funções ecológicas, sendo fonte de alimento para outros invertebrados, anfíbios, aves e peixes (Maltchick et al. 2010). Ao explorarem uma ampla faixa de recursos alimentares, podem auxiliar na ciclagem de nutrientes, no consumo da vegetação aquática e no controle de populações de peixes (Pereira et al. 2001). Os moluscos límnicos dependem diretamente dos parâmetros bióticos (ex: macroalgas, microalgas e outras comunidades de animais) e abióticos (ex: temperatura, profundidade, oxigênio) (Petsch et al. 2013). Entre os principais fatores abióticos que afetam as comunidades de moluscos, destacam-se o oxigênio, que é o principal fator limitante (Vazzoler 1981), e a temperatura, que interferem na quantidade de matéria orgânica no ambiente, na alimentação e reprodução dos moluscos (Mota 2012); o pH, que é um fator extremamente importante, pois influencia na formação das conchas, onde em meio muito ácidos essas estruturas calcárias são impedidas de serem formadas (Souza et al. 1998); e a luminosidade e profundidade que atuam como condição ao estabelecimento de macrófitas aquáticas que por fornecer alimentos e disponibilidade de refúgios contra predadores (Esteves 1998, Jorcin \& Nogueira 2008).

O gastrópode Biomphalaria straminea, destacase entre os moluscos límnicos devido a sua ampla distribuição geográfica, ocorrendo desde a Costa Rica até a Argentina (Paraense 1977). No Brasil está presente em um grande número de municípios brasileiros (1.327 municípios), distribuídos por 24 Estados, além do Distrito Federal, com exceção dos estados do Amapá e Rondônia (Ministério da Saúde 2008). Sua ampla distribuição pode ser associada ao seu processo de dispersão por animais, pela correnteza ou por ação humana (Kappes \& Haase, 2012).

Portanto, é notório a importância dos parâmetros abióticos para reprodução, alimentação, e crescimento das espécies de moluscos, podendo limitar assim sua composição e abundância no ambiente. Diante disto, no presente estudo avaliamos as variáveis abióticas que afetam a abundância de Biomphalaria straminea em uma lagoa temporária no semiárido de Pernambuco.

\section{MATERIAL E MÉTODOS}

O Parque Estadual Mata da Pimenteira (PEMP; $\left.07^{\circ} 53^{\prime} 48,96^{\prime \prime} \mathrm{S}, \quad 38^{\circ} 18^{\prime} 14,30^{\prime \prime} \mathrm{W}\right)$ está situado no município de Serra Talhada, Estado de Pernambuco, Brasil, a $450 \mathrm{Km}$ da capital Recife. Possui uma área de 887,24 ha e está localizado na unidade de paisagem depressão sertaneja, uma paisagem típica do semiárido nordestino, com relevo predominantemente suave e ondulado, baixa pluviosidade e vegetação predominante de Caatinga (Sá et al. 2003). De acordo com Diniz et al. (2013), o PEMP possui duas lagoas e um riacho temporários, além de possuir uma rica diversidade de fauna e flora.

O estudo foi desenvolvido na Lagoa II da Pimenteira (Figura 1), que consiste em um corpo d'água que durante a cheia pode atingir uma área de aproximadamente 0,283 ha e caracteriza-se por apresentar normalmente um período de seca por ano (Diniz et al. 2013). Foram selecionados cinco pontos de amostragem na lagoa, sendo quatro nas margens e um no centro da lagoa (Figura 1). $\mathrm{O}$ 


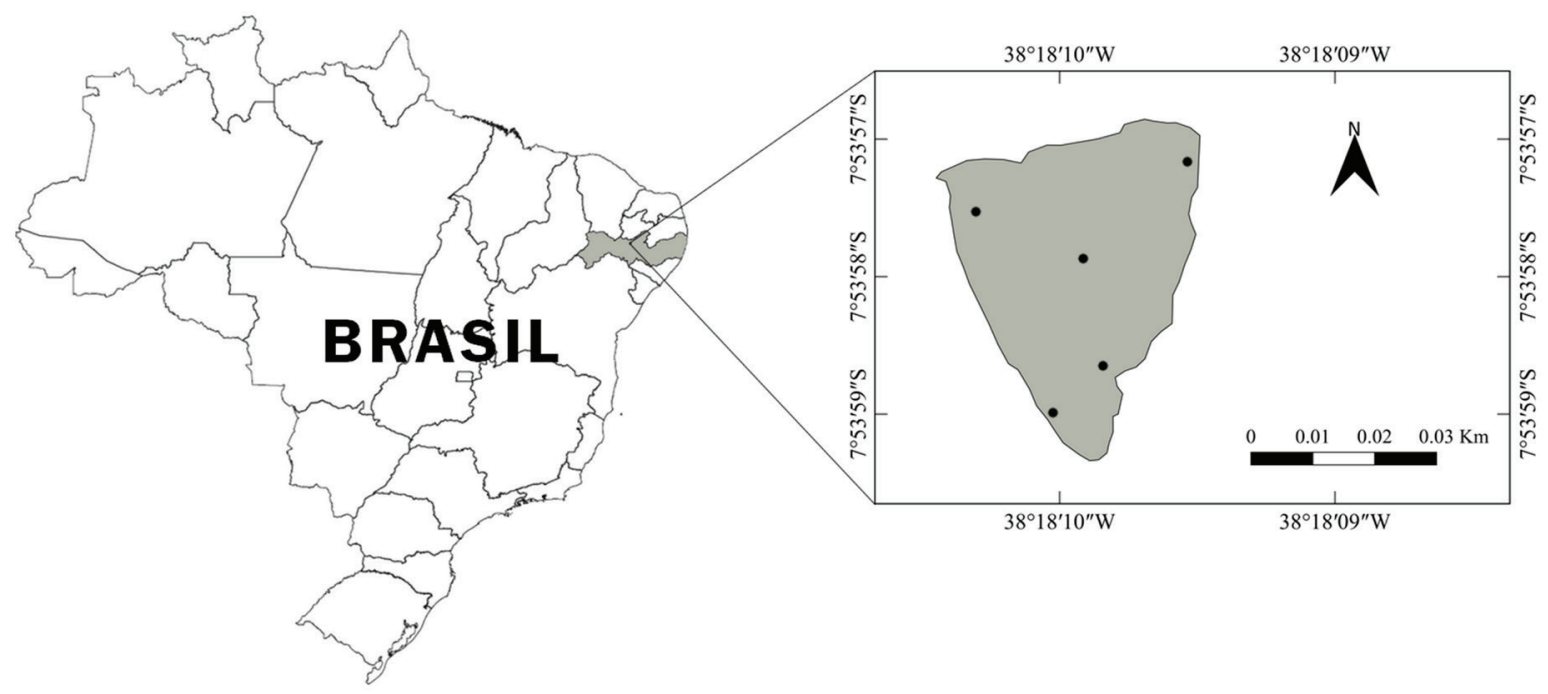

Figura 1. Localização da Lagoa II no Parque Estadual Mata da Pimenteira (Serra Talhada, Pernambuco, Brasil). No detalhe, esquema da distribuição dos cinco pontos de amostragem no interior da lagoa.

Figure 1. Location of the Lagoa II in the Mata da Pimenteira State Park (Serra Talhada, Pernambuco, Brazil). In detail, scheme of the distribution of the five sampling points inside the lagoon.

período de estudo foi de outubro/2014 ajulho/2015. Os dados referentes às variáveis limnológicas (temperatura da água em ${ }^{\circ} \mathrm{C}, \mathrm{pH}$, oxigênio dissolvido em $\mathrm{mg} / \mathrm{L}$ e condutividade elétrica em $\mathrm{nS} / \mathrm{cm}$ ) foram mensurados mensalmente em cada um dos cinco pontos de amostragem utilizando uma sonda de multiparâmetro, modelo Hanna HI9828. Os parâmetros físico-químicos de abril/2015 não foram mensurados devido a lagoa encontrar-se seca.

Para a amostragem dos gastrópodes, em cada um dos pontos de amostragem foi feito um arrasto nos meses de outubro e dezembro de 2014 e fevereiro, maio e julho de 2015 com auxílio de puçá, com malha de $2 \mathrm{~mm}$ (Nascimento-Filho et al. 2014). Cada arrasto foi realizado de forma manual e com duração estabelecida de $1 \mathrm{~min}$. Em seguida, o material foi acondicionado em sacos plásticos, com água e sedimento do ambiente, etiquetados, e encaminhados para o laboratório para posterior análise. No laboratório, os indivíduos foram crioanestesiados com choque térmico. Durante a triagem em laboratório, as amostras foram lavadas com água corrente em quatro peneiras biológicas com malhas de $2 \mathrm{~mm}, 1 \mathrm{~mm}, 0,5 \mathrm{~mm}$ e $0,25 \mathrm{~mm}$ cada, para facilitar a separação dos gastrópodes do substrato (Nascimento-Filho et al. 2014). Os gastrópodes retidos nas peneiras foram armazenados em potes plásticos com álcool a $70 \%$ e etiquetados. A identificação dos táxons foi realizada por chave de identificação de Barbosa (1995) e Simone (2006), observandose a morfologia das conchas com auxílio de estereomicroscópio.

Os dados obtidos foram inicialmente tratados quanto à riqueza de espécies e ao índice de abundância (ind./min). O método quantitativo aplicado para avaliar a abundância destes organismos foi adaptado de Olivier \& Schneiderman (1956), caracterizado por captura de indivíduos em determinado tempo. O índice de abundância de cada local de coleta foi estimado por meio da seguinte fórmula: índice de abundância $=\mathrm{N}^{\circ}$ de moluscos coletados por mês I tempo total de arrasto. O tempo total de arrasto é dado pelo tempo de arrasto $\mathrm{x} \mathrm{N}^{\circ}$ de pontos de coleta.

Em cada mês foi feita a média entre os cinco pontos amostrados para os fatores abióticos (temperatura da água, oxigênio dissolvido, $\mathrm{pH}$ e 
condutividade elétrica) e testada a normalidade dos dados por Shapiro-Wilk (Zar 1999), e então realizado regressão linear entre cada variável com o índice de abundância de B. straminea.

\section{RESULTADOS}

A temperatura média da água foi $26,6^{\circ} \mathrm{C}$, com valor máximo $\left(28,7^{\circ} \mathrm{C}\right)$ no mês de março $/ 2015$ e mínimo $\left(24,9^{\circ} \mathrm{C}\right)$ no mês de julho/2015 (Figura 2a). A variação do oxigênio foi de 4,96 a 6,99 mg/L, exceto nos meses de dezembro/2014 e maio/2015, cujos valores foram menores do que $3 \mathrm{mg} / \mathrm{L}$ (Figura $2 \mathrm{~b}$ ). $\mathrm{O}$ valor do $\mathrm{pH}$ foi máximo $(8,7)$ em fevereiro/2015 e mínimo $(4,3)$ em dezembro/2014 (Figura 2c). A lagoa estudada apresentou baixa condutividade elétrica com média de 0,26 nS/cm (Figura 2d).

Foram coletados 199 indivíduos de gastrópodes, representados por duas espécies, sendo elas: Biomphalaria straminea (Dunker, 1848) (Basommatophora: Planorbidae) com 97,99\% dos indivíduos e Pomacea lineata (Spix, 1827) (Architaenioglossa: Ampullariidae) com 2,01\% dos indivíduos (Figura 3). A maioria dos gastrópodes presentes na lagoa apresentaram conchas menores que $2 \mathrm{~mm}(85,43 \%)$.

Em relação ao índice de abundância mensal, os meses de julho e fevereiro/2015 apresentaram maiores abundâncias relativas, com 16,4 e 11,4 ind./min, respectivamente (Tabela 1). O teste de normalidade mostrou que os dados possuem distribuição normal $(\mathrm{p}>0.05)$. O índice de abundância mensal foi relacionado com a temperatura da água $\left(\mathrm{R}^{2}=0,838, \mathrm{p}=0,027, \mathrm{gl}=4\right.$; Figura 4a) e com o oxigênio dissolvido $\left(\mathrm{R}^{2}=0,731\right.$, $\mathrm{p}=0,044, \mathrm{gl}=4$; Figura $4 \mathrm{~b})$. Para o $\mathrm{pH}\left(\mathrm{R}^{2}=0,062, \mathrm{p}\right.$ $=0,685, \mathrm{gl}=4$; Figura $4 \mathrm{c}$ ) e a condutividade elétrica $\left(\mathrm{R}^{2}=0,001, \mathrm{p}=0,99, \mathrm{gl}=4\right.$; Figura $\left.4 \mathrm{~d}\right)$ não houve relação significativa.
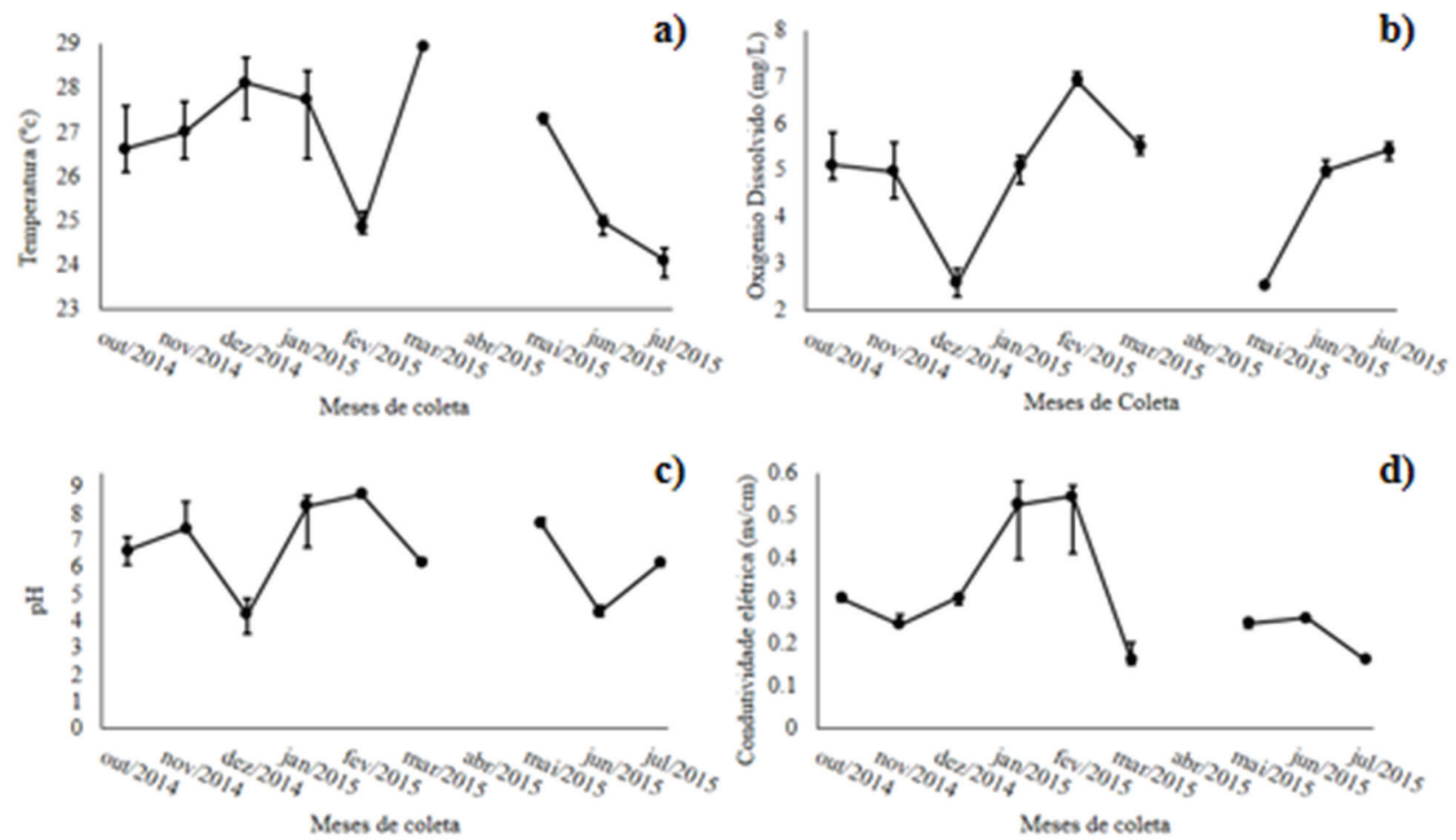

Figura 2. Variação dos parâmetros abióticos na Lagoa II do Parque Estadual da Mata da Pimenteira (Serra Talhada, Pernambuco, Brasil). Os círculos indicam os valores médios por mês e as barras a amplitude de variação. a) Temperatura da água $\left({ }^{\circ} \mathrm{C}\right)$; b) Oxigênio dissolvido $(\mathrm{mg} / \mathrm{L})$; c) $\mathrm{pH}$; d) Condutividade elétrica (nS/ $\mathrm{cm})$.

Figure 2. Variation of abiotic parameters in Lagoa II in the Mata da Pimenteira State Park (Serra Talhada, Pernambuco, Brazil). Circles indicate the mean values per month and bars indicate range variation. a) Temperature $\left.\left({ }^{\circ} \mathrm{C}\right) ; b\right)$ Dissolved oxygen ( $\left.\left.\mathrm{mg} / \mathrm{L}\right) ; \mathrm{c}\right) \mathrm{pH}$; d) Electric conductivity ( $\left.\mathrm{nS} / \mathrm{cm}\right)$. 

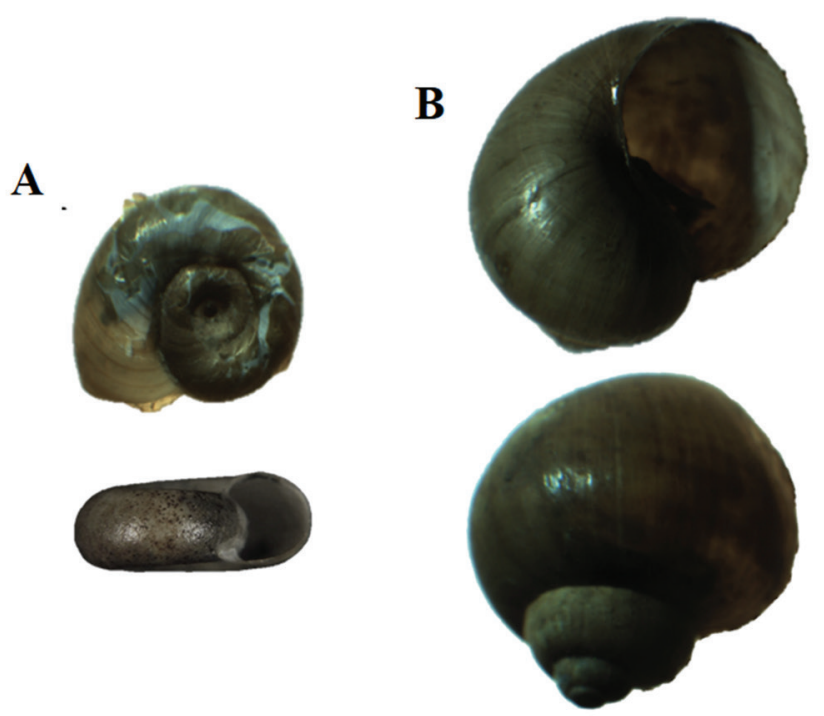

\section{$1 \mathrm{~cm}$}

Figura 3. Espécies de gastrópodes encontradas na Lagoa II do Parque Estadual da Mata da Pimenteira (Serra Talhada, Pernambuco, Brasil). a) Biomphalaria straminea (Basommatophora: Planorbidae); b) Pomacea lineata (Architaenioglossa: Ampullariidae).

Figure 3. Gastropods species found in Lagoa II in the Mata da Pimenteira State Park (Serra Talhada, Pernambuco, Brazil). a) Biomphalaria straminea (Basommatophora: Planorbidae); b) Pomacea lineata (Architaenioglossa: Ampullariidae).

Tabela 1. Quantidade de gastrópodes por espécie capturados na Lagoa II do Parque Estadual da Mata da Pimenteira (Serra Talhada, Pernambuco, Brasil) no período entre outubro/2014 e julho/2015.

Table 1. Number of gastropods per species collected in Lagoa II in the Mata da Pimenteira State Park (Serra Talhada, Pernambuco, Brazil) between October/2014 and July/2015.

\begin{tabular}{lccccccc}
\hline \multicolumn{1}{c}{ Espécie } & out/2014 & dez/2014 & fev/2015 & mai/2015 & jul/2015 & Total \\
\hline Biomphalaria straminea & 44 & 9 & 57 & 3 & 82 & 195 \\
Pomacea lineata & 1 & 0 & 0 & 0 & 3 & 4 \\
\hline & Total & 45 & 9 & 57 & 3 & 85 & 199 \\
Abundância de B. straminea (ind./min) & 8,8 & 1,8 & 11,4 & 0,6 & 16,4 & - \\
\hline
\end{tabular}

\section{DISCUSSÃO}

A predominância de $B$. straminea sobre P. lineata pode estar relacionada à biologia reprodutiva das espécies, visto que $B$. straminea apresenta desovas de 65 a 71 ovos por indivíduo, com eclosão normalmente sete dias após postura (Costa et al. 2004). Já P. lineata possui desova de 20 a 80 ovos e um período de incubação mais longo que a B. straminea, tendo duração de 15 a 23 dias
(Kretzschmar \& Heckman 1995). Entretanto, a desova de B. straminea ocorre normalmente nos períodos chuvosos, que é menos comum na região do estudo, já que a lagoa está inserida na Caatinga (Andrade \& Carvalho 1979, Souza \& Lima 1990, Costa et al. 2004). Em relação ao tamanho das conchas dos indivíduos, de acordo com Souza \& Lima (1990), esse elevado número de gastrópodes com maior tamanho de concha pode estar vinculado ao crescimento rápido das espécies registradas e ao 


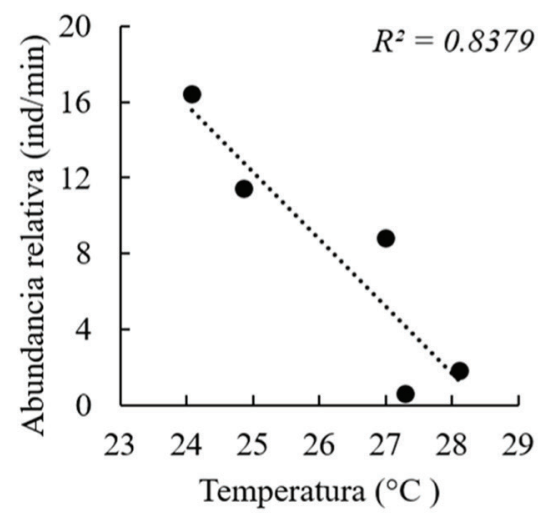

a)

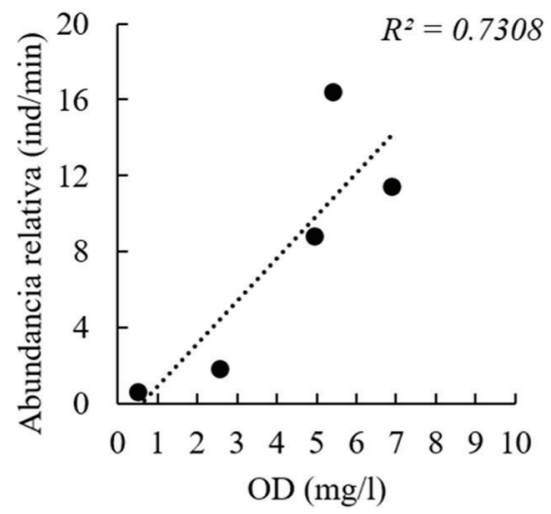

b)

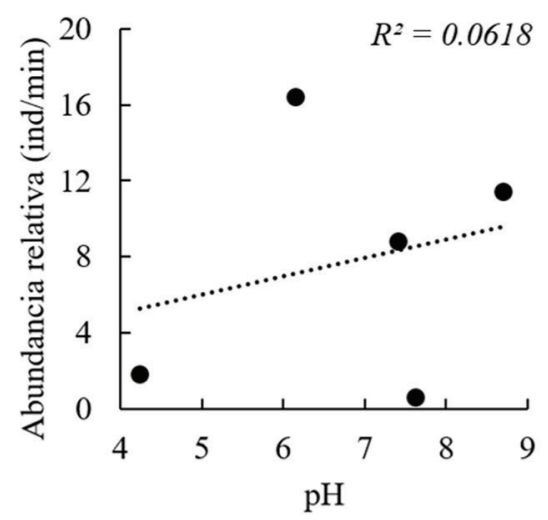

c)

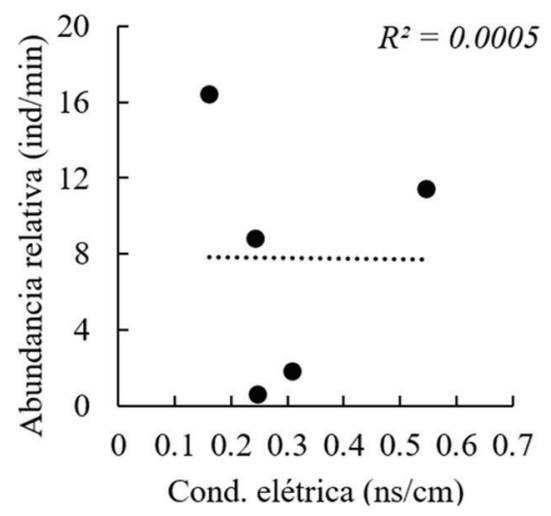

Figura 4. Regressão linear entre os parâmetros abióticos e o índice de abundância de Biomphalaria straminea na lagoa II do Parque Estadual da Mata da Pimenteira (Serra Talhada, Pernambuco, Brasil). $\mathrm{R}^{2}$ = coeficiente de determinação da regressão linear. a) Temperatura da água $\left({ }^{\circ} \mathrm{C}\right)$; b) Oxigênio dissolvido $(\mathrm{mg} / \mathrm{L})$; c) $\mathrm{pH}$; d) Condutividade elétrica $(\mathrm{nS} / \mathrm{cm})$.

Figure 4. Linear regression between of abiotic parameters and abundance of Biomphalaria straminea in Lagoa II in the Mata da Pimenteira State Park (Serra Talhada, Pernambuco, Brazil). $R^{2}=$ linear regression coefficient. a) Temperature $\left.\left({ }^{\circ} \mathrm{C}\right) ; b\right)$ Dissolved oxygen $\left.(\mathrm{mg} / \mathrm{L}) ; \mathrm{c}\right) \mathrm{pH}$; d) Electric conductivity $(\mathrm{nS} / \mathrm{cm})$.

fato delas poderem atingir a maturação sexual em torno de 30 dias.

A variação nos valores de temperatura observada no corpo d’água está na faixa considerada favorável para que gastrópodes límnicos estabeleçam suas atividades de alimentação e reprodução, uma vez que o limite ideal para estes gastrópodes varia entre $18^{\circ} \mathrm{C}$ a $25^{\circ} \mathrm{C}$ (Barbosa 1995). O índice de abundância mensal de $B$. straminea foi negativamente relacionado com a temperatura, ou seja, à medida que a temperatura diminui, maior o índice de abundância. Portanto, é possível inferir que a temperatura é um fator determinante na abundância desses gastrópodes.

Já os valores de oxigênio dissolvido foram relacionados com o índice de abundância, sugerindo que, quanto maior a dissolução de oxigênio na água, maior a abundância de $B$. straminea. Mota et al. (2012), ao realizarem uma análise similar, também encontraram um valor de correlação positiva próximo ao encontrado no PEMP no presente estudo, mostrando, assim, que esse parâmetro também influencia na abundância.

Em relação ao pH, Pieri (2008) afirma que os gastrópodes se desenvolvem melhor em ambientes com pH próximo a 6,0 e 8,0 , sendo que apenas nos meses de dezembro/2014 e março/2015 os valores ficaram abaixo dessa faixa estabelecida. Esse parâmetro é um fator extremamente importante, pois as estruturas calcárias, material essencial para formação de conchas, são impedidas de serem formadas em um meio muito ácido devido às reações químicas, limitando o desenvolvimento desses animais em determinados locais (Souza et 
al. 1998). Na lagoa estudada não foi encontrada uma relação entre o pH e o índice de abundância, contudo em estudos similares foram encontradas uma correlação positiva, tais como Callisto et al. (2005), que estudaram a malacofauna no reservatório de Ibirité em Belo Horizonte, e Souza et al. (2008) na bacia do Alto Rio Paraná.

Já a condutividade elétrica da água pode variar em função direta da quantidade de sais dissolvidos (íons) e da temperatura (Arrignon 1979). Esses valores de condutividade variam ao longo do ano em função das chuvas e da evaporação, que alteram a concentração dos sais na água (Madsen 1985). Este fator não foi significativamente relacionado com a abundância dos gastrópodes encontrados.

Concluindo, a temperatura da água e o oxigênio dissolvido foram os únicos parâmetros, no presente estudo, que apresentaram relações significativas com a abundância de B. straminea que ocorre na lagoa II, mostrando assim a importância desses parâmetros como reguladores da abundância deste gastrópode.

\section{AGRADECIMENTOS}

Ao Programa de Educação Tutorial (PET) do curso de Engenharia de Pesca da UFRPE-UAST, pela bolsa concedida ao primeiro autor durante o estudo desenvolvido, aos colegas que fazem parte do Laboratório de Bentos da UFRPE-UAST, a Thaisy Emmanuelle da Silva e Patrick Anderson Moura pelo auxilio nas coletadas e triagem do material biológico do presente estudo e a Carlos Yure Oliveira pelas traduções e correções.

\section{REFERÊNCIAS}

Andrade, R. M., \& Carvalho, O. S. 1979. Colonização de Pomacea haustrum (Reeve, 1856) em localidade com esquistossomose mansoni: Baldim, MG (Brasil). (Prosobranchia, Pilidae). Revista de Saúde Pública, 13(2), 92-107. DOI: 10.1590/S0034-89101979000200005

Aranguren-Riaño, N., Guisande, C., \& Ospina, R. 2011. Factors controlling crustacean zooplankton species richness in Neotropical lakes. Journal of Plankton Research, 33(8), 12951303. DOI: $10.1093 /$ plankt/fbr028
Arrignon, J. 1979. Ecologia y piscicultura de aguas dulces. Ed. Mundi Prensa, Espanha. p. 365.

Barbosa, F. S. 1995. Tópicos em Malacologia Médica. Rio de Janeiro: Editora Fiocruz: p. 314.

Callisto, M., Moreno, P., Gonçalves, J. F. J. R., Ferreira, W. R., \& Gomes, C. L. Z. 2005. Malacological assessment and natural infestation of Biomphalaria straminea (Dunker, 1848) by Schistosoma mansoni (Sambon, 1907) and Chaetogaster limnaei (K. Von Baer, 1827) in an urban eutrophic watershed. Brazilian Journal of Biology, 65(2), 217-28. DOI: 10.1590/S151969842005000200005

Cardoso, M. M. L., Souza, J. E. R. T., Crispim, M. C., \& Siqueira, R. 2012. Diversidade de peixes de um rio intermitente do semiárido paraibano, Brasil. Biotemas, 25(3), 161-171. DOI: 10.5007/2175-7925.2012v25n3p161

Coelho, L. H. L. 2007. Lymnaea columella (Pulmonata: Lymnaeidae): dinâmica de populações em Itajubá, Minas Gerais e suscetibilidade à infecção por Fasciola hepatica (Digenea: Fasciolidae) em associações simpátricas e alopátricas entre parasito e hospedeiro. Tese de doutorado. Departamento de Parasitologia da Universidade Federal de Minas Gerais. p. 112.

Costa, M. J. F., Grault, C. E., \& Confalonieri, U. E. 2004. Comparative study of the fecundity and fertility of Biomphalaria glabrata (Say, 1818) and Biomphalaria straminea (Dunker, 1848) in a laboratory through self-fertilization and crossfertilization. Revista do Instituto de Medicina Tropical de São Paulo, 46(3), 157-163. DOI: 10.1590/S0036-46652004000300007

Diniz, L. P., Elmoor-Loureiro, L. M., Almeida, A. V. L. S., \& Júnior, M. M. 2013. Cladocera (Crustacea, Branchiopoda) of a temporary shallow pond in the Caatinga of Pernambuco, Brasil. Nauplius, 21(1), 65-78. DOI: 10.1590/S010464972013000100008

Esteves, F. A. 1998. Fundamentos de Limnologia. Rio de Janeiro: Interciência/FINEP: p. 602.

Jorcin, A., \& Nogueira, M. G. 2008. Benthic macroinvertebrates in the Paranapanema reservoir cascade (southeast Brazil). Brazilian Journal of Biology, 68(4), 1013-1024. DOI: 10.1590/S1519-69842008000500009

Kappes, H., \& Haase, P. 2012. Slow, but steady: dispersal of freshwater molluscs. Aquatic 
Sciences, 74(1), 1-14. DOI: 10.1007/s00027-0110187-6

Kretzschmar, A. U., \& Heckman, C. 1995. Estratégias de sobrevivência das espécies de Ampullariidae (Mollusca, Gastropoda) durante mudanças das condições ambientais extremas do ciclo sazonal sob o clima tropical úmido-e-seco. Acta Limnologica Brosiliensia, 7, 60-66.

Madsen, H. 1985. Ecology and control of African freshwater pulmonate snails. Part I: Life cycle and methodology. Charlottenlund: Danish Bilharziasis Laboratory: p. 36.

Maltchik, L. 2000. Lagoas temporárias do semiárido. Ciência Hoje, 28(167), 67-70.

Maltchik, L., Stenert, C., \& Kotzian, C. B. D. 2010. Responses of freshwater molluscs to environmental factors in Southern Brazil wetlands. Brazilian Journal the Biology, 70(3), 473-482. DOI: 10.1590/S151969842010005000003

Ministério da Saúde. 2008. Vigilância e controle de moluscos de importância epidemiológica - Diretrizes técnicas: Programa de Vigilância e Controle da Esquistossomose (PCE) - 2. ed. Brasília. p. 178.

Mota, D. J. G., Moraes, J., Nascimento, C., Kawano, T., \& Pinto, P. L. S. 2012. Malacofauna límnica em pesqueiro de Itapecerica da Serra, São Paulo, Brasil: risco potencial na transmissão de helmintoses. Boletim do Instituto de Pesca, 38(4), 297-312.

Mugnai, R., Oliveira, R. B., Carvalho, L., \& Batista, D. F. 2010. Adaptation of the Indice Biotico Esteso (IBE) for water quality assessment in rivers of Serra do Mar, Rio de Janeiro State, Brazil. Tropical Zoology, 21, 57-74.

Nascimento Filho, S. L., Viana, G. F. S., \& Gomes, R. L. M. 2014. Inventário da malacofauna límnica de três grandes reservatórios do sertão de Pernambuco, Brasil. Scientia Plena, 10(11), 1-7.

Olivier, L., \& Scheneiderman, M. 1956. A method for estimating the density of aquatic snail populations. Experimental Parasitology, 5(2), 109-117. DOI: 10.1016/0014-4894(56)90008-X

Paraense, W. L. 1977. Distribuição geográfica dos vetores da xistosomose no Nordeste do Brasil. In: P. A. Machado (Ed.), Painel programa especial de controle de esquistossomose. pp. 47-51. Brasília: Ministério da Saúde.

Petsch, D. K., Pinha, G. D., Ragonha, F. H., \& Takeda,
A. M. 2013. Influência dos fatores ambientais sobre a distribuição da comunidade de invertebrados bentônicos em canais de uma planície de inundação neotropomunidade de invertebrados bentônicos em canais secundários e principal de uma planície de inundação neotropical. Biotemas, 26(3), 127138. DOI: $10.5007 / 2175-7925.2013 v 26 n 3 p 127$

Pereira, D., Inda, L. A., Consoni, J. M., \& Konrad, H. G. 2001. Composição e abundância de espécies de moluscos do bento marginal da microbacia do arroio Capivara, Triunfo, RS, Brasil. Biociências, 9(1), 3-20.

Pieri, O. S. 2008. Diretrizes técnicas: Programa de Vigilância e Controle da Esquistossomose (PCE) - 2. Aspectos ecológicos. Vigilância e Controle de Gastrópodes de Importância Epidemiológica. Brasília: Ministério da Saúde: p. 129.

Pineda, M. D., \& Schäfer, A. 1987. Adequação, critérios e métodos de avaliação de águas superficiais baseada no estudo ecológico do rio Gravataí. Ciência e Cultura, 39(2), 198-206.

Rocha, O. 2003. Águas Doces (Versão Preliminar). Avaliação do estado do conhecimento da diversidade biológica do Brasil. Brasília: COBIO / MMA-GTB/CNPq- NEPAM/UNICAMP: p. 12.

Sa, I. B., Riché, G. R., \& Fotius, G. A. 2003. As paisagens e o processo de degradação do semiárido nordestino. Embrapa Semiárido: p. 36.

Simões, N. R., Sonoda, S. L., \& Ribeiro, S. M. M. S. 2008. Spatial and seasonal variation of microcrustaceans (Cladocera and Copepoda) in intermittent rivers in the Jequiezinho River Hydrographic Basin, in the Neotropical semiarid. Acta Limnologica Brasiliensia, 20(3), 197-204.

Simões, R. I. 2002. Comunidade de gastrópodes bentônicos na área de abrangência da usina hidrelétrica de Dona Francisca, Rio Jacuí, Rio Grande do Sul, Brasil: fase de pré e pósenchimento. Dissertação de mestrado. Departamento de Biologia Animal da Universidade Federal do Rio Grande do Sul. p. 217.

Simone, L. R. L. 2006. Land and freshwater molluscs of Brazil. São Paulo: FAPESP: p. 390.

Souza, C. P., \& Lima, L. C. 1990. Gastrópodes de interesse parasitológico do Brasil. Belo Horizonte: Editora Fiocruz: p. 72.

Souza, C. P., Drummond, S. C., Silva, C. J. E., 
Queiroz, L. A., Guimarães, C.T., \& Rocha, R. S. 1998. Investigação sobre a transmissão da esquistossomose no complexo turístico da Serra do Cipó, MG. Informe Epidemiológico do Sus, 6(4), 43-51. DOI: 10.5123/S010416731998000400005

Souza, G. T. R., Machado, M. H., Dias, M. L. G. G., Yamada, F. H., Pagotto, J. P. A, \& Pavanelli, G. C. 2008. Composição e sazonalidade dos gastrópodes do alto rio Paraná, Brasil, e sua potencialidade como hospedeiros intermediários de digenéticos. Acta Scientiarum Biological Sciences, 30(2), 309-314. DOI: 10.4025/actascibiolsci.v30i3.5018

Tundisi, J. G., \& Matsumura-Tundisi, T. 2008. Limnologia. São Paulo: Oficinas de Textos: p. 631.

Vazzoler, A. E. A. M. 1981. Manual de métodos para estudos biológicos de populações de peixes, reprodução e crescimento. Brasília: CNPq: p. 108.

Vidigal, T. H. D. A., Marques, M. M. G. S. M., Lima, H. P., \& Barbosa, F. A. R. 2005. Gastrópodes e bivalves límnicos do trecho médio da bacia do Rio Doce, Minas Gerais, Brasil. Lundiana, 6, 67-76.

Zar, J. H. 1999. Biostatistical Analysis. 4th. ed. New Jersey: Prentice Hall: p. 196.

Submetido: $27 / 03 / 2018$

Aceito: $28 / 10 / 2018$

Publicado online: 28/10/2018

Editor Associado: Fábio Maffei 